\title{
Internal Reliability and Validity of Copenhagen Burnout Inventory and Oldenburg Burnout Inventory Compared with Maslach Burnout Inventory among Nigerian Resident Doctors: A Pilot Study
}

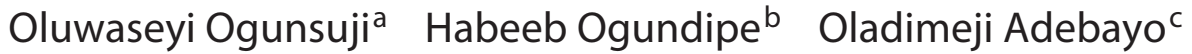 \\ Tolulope Oladehin $^{d}$ Sebastine Oiwoh $^{\mathrm{e}}$ Olufemi Obafemi ${ }^{f}$ \\ Oluwafunmilayo Soneye ${ }^{\text {b }}$ Oyinkansola Agaja $^{d}$ Okhuaihesuyi Uyilawa ${ }^{9}$ \\ Oluwafemi Efuntoye ${ }^{c}$ Taiwo Alatishe ${ }^{\text {h }}$ Adedayo Williams $^{i}$ \\ Olayinka llesanmi ${ }^{j}$ Olayinka Atilolak
}

\begin{abstract}
aDepartment of Periodontology \& Community Dentistry, University College Hospital, Ibadan, Nigeria; ${ }^{b}$ Department of Surgery, University College Hospital, Ibadan, Nigeria; 'Department of Medicine, University College Hospital,

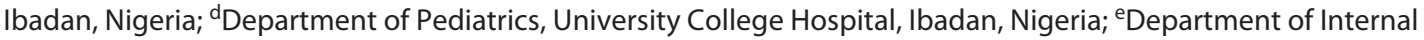
Medicine, Irrua Specialist Teaching Hospital, Irrua, Nigeria; 'Department of Microbiology, University College Hospital, Ibadan, Nigeria; ${ }^{9}$ Department of Orthopedics and Trauma, Delta State University Teaching Hospital, Ogharra, Nigeria; ' Department of Psychiatry, Ladoke Akintola University of Technology Teaching Hospital, Ogbomoso, Nigeria; 'Department of Family Medicine, University College Hospital, Ibadan, Nigeria; ${ }^{\text {DDepartment }}$ of Community Medicine, University of Ibadan/University College Hospital, Ibadan, Nigeria; ${ }^{2}$ Department of Behavioural Medicine, Lagos State University, College of Medicine, Ikeja, Nigeria
\end{abstract}

\section{Keywords}

Burnout · Resident doctors · Validation · Reliability

\begin{abstract}
Introduction: Copyrighted Maslach Burnout Inventory (MBI) is perhaps the most widely used and validated tool in assessing burnout among different occupations and health care professionals compared to the free to use Copenhagen Burnout Inventory $(\mathrm{CBI})$ and Oldenburg Burnout Inventory (OLBI). This study aimed to determine the reliability and validity of these tools in comparison with $\mathrm{MBI}$ among a subset of Nigerian resident doctors. Methods: A cross-sectional survey with reliability of the burnout scales calculated using Cronbach's alpha. Construct validity was assessed by princi-
\end{abstract}

karger@karger.com www.karger.com/dmj

Karger $\stackrel{\text { ' }}{5}$

GOPEN ACCESS
(C) 2022 The Author(s)

Published by S. Karger AG, Basel

This is an Open Access article licensed under the Creative Commons Attribution-NonCommercial-4.0 International License (CC BY-NC) (http://www.karger.com/Services/OpenAccessLicense), applicable to the online version of the article only. Usage and distribution for commercial purposes requires written permission. pal component analysis and correlating dimensions within each burnout tool with one another using Pearson's correlation coefficient. The criterion validity of each dimension was assessed for the ability of independent variables to predict their scores using multiple linear regression. Results: Copenhagen Personal Burnout dimension had the highest Cronbach's alpha score of 0.91. MBI-Emotional Exhaustion had the highest correlations with Copenhagen Work-related, Copenhagen Personal-related, and Oldenburg Exhaustion burnout dimensions. Only the multiple regression models for Copenhagen personal $(p=0.04)$ and work-related $(p=$ 0.02 ) burnout dimensions were significant, with the specialty of the residents being the significant independent variable in both models. Conclusion: CBI and OLBI have high internal consistency and reliability among the subset of res- 
ident doctors recruited into this study, $\mathrm{CBI}$ dimensions had the best predictive and construct validity and can be used as valid alternative to $\mathrm{MBI}$.

(c) 2022 The Author(s).

Published by S. Karger AG, Basel

\section{Introduction}

The World Health Organization conceptualizes burnout as a syndrome resulting from chronic workplace stress that has not been successfully managed and is characterized in 3 dimensions: feelings of energy depletion or exhaustion, increased mental distance from one's job and/or feelings of negativism or cynicism related to one's job, and reduced professional efficacy [1]. The consequences of burnout are potentially serious for the workers, their clients, and the society with which they interact [2]. Investigating this workplace problem is essential to formulating strategies to help reduce and cope with burnout and its effects. Choosing a reliable and valid tool for this purpose is crucial to obtaining accurate and relevant data. Several tools are being used to measure burnout; the popular ones include the Maslach Burnout Inventory (MBI), Oldenburg Burnout Inventory (OLBI), and Copenhagen Burnout Inventory (CBI), among others. Though there is no consensus as to the best measure of burnout, MBI is seen as the most reliable measure for professionals who work with people $[3,4]$.

MBI was first developed by Maslach [2] and is perhaps the most widely used and validated tool in assessing burnout in research undertakings across different occupations, cultures, and countries [5-13]. In sub-Saharan Africa, most researchers use the MBI, and the trend is adopted by researchers evaluating burnout among health workers in Nigeria, although most of these studies utilized small sample sizes [14-20]. MBI-Human Services Survey (MBI-HSS) used for health care professionals' costs 2.50 USD to purchase a license to reproduce and administer to one individual. This might pose huge financial burden on investigators/scientists in resource-constrained environments especially in situations where the population to be investigated is large and research funding is poor. The CBI and OLBI are currently available nocost alternatives [21-23].

The OLBI first developed by Demerouti et al. in 2001 [24] has been validated for use by employees in different occupational fields including health workers in different parts of the world, likewise CBI, which was first developed by Kristensen et al. in 2005 [21,25-31]. Their use in Africa is however limited and evidence of their validity for use in this environment is equally limited in available literature. A systematic review of burnout among health care workers in sub-Saharan Africa showed that OLBI and $\mathrm{CBI}$ are sparingly used as the burnout inventories in Africa [17]. A recent cross-sectional study by Nwosu et al. [32] on-physician's burnout across 5 tertiary hospitals in Nigeria used OLBI to measure burnout. They reported higher mean burnout score among resident doctors in comparison to medical consultants for both exhaustion and disengagement dimensions of OLBI. The mean burnout score among residents was similar to that for interns and medical officers in both dimensions of OLBI. Arguably, one possible reason for the limited use of the CBI and OLBI might be because these two burnout inventories are yet to be validated within the sub-Saharan African population context.

Evidently, MBIs role as a validated instrument in assessing burnout is incontrovertible, thus it serves as a standard for which the reliability and validity of other burnout tools can be assessed, as will be done for CBI and OLBI in evaluating burnout among early career doctors in Nigeria, in this study. Hence, our study set out to determine the internal reliability of OLBI and CBI compared with MBI as the gold-standard burnout measure among a subset of resident doctors in Nigeria. We hypothesized that OLBI and CBI will have internal reliability that is comparable with that of MBI.

\section{Methods}

Study Design, Location, and Participants

This was a cross-sectional pilot study, a part of the ongoing challenges of residency training in Nigeria (CHARTING phase I \& II) study among early career doctors, the protocols of which have been described in previous publications $[33,34]$. The study was conducted within a month, from 1st to 30th of September 2020 among resident doctors who were either senior registrars or registrars. Study participants were recruited from 2 tertiary hospitals, namely, University College Hospital (UCH), Ibadan and Ladoke Akintola University of Technology Teaching Hospital Ogbomosho (LTH Ogbomosho). To be included in the study, a resident doctor ought to have been employed for at least 3 months in his/ her hospital. A total of 100 resident doctors were recruited, 80 from $\mathrm{UCH}$ and 20 from LTH Ogbomosho through a random probability sampling technique. Informed consent was also gotten from all the study participants.

\section{Sampling Procedure}

The participants were recruited and classified into groups (physicians and surgeons) based on the two West African Colleges of postgraduate medical training (West African College of Physicians (WACP) or West African College of Surgeons (WACS) based on the institutional department they are currently training
Ogunsuji et al. 
Table 1. Sociodemographic characteristics of participants

\begin{tabular}{ll}
\hline Sociodemographic & Median (IQR) \\
\hline Age & $35(32.5-38.5)$ \\
Years on current job, $n$ & $4(1-7)$ \\
Years since graduation, $n$ & $10(7-11)$ \\
\hline & Frequency (\%) \\
\hline Specialty categorization & \\
Medical & $48(48.0)$ \\
Surgical & $52(52.0)$ \\
Cadre & $46(46.0)$ \\
Registrar & $54(54.0)$ \\
Senior registrar & $27(27.0)$ \\
Marital status & $72(72.0)$ \\
Single & $1(1.0)$ \\
Married & \\
Divorced & $69(69.0)$ \\
Gender & $31(31.0)$ \\
$\quad$ Male &
\end{tabular}

and working [35]. Each of the colleges has various faculties (WACP $=6$ faculties and WACS $=8$ ). To recruit participants in $\mathrm{UCH}$, we selected 2 faculties using simple random sampling from each of the groups. In the WACS, Faculty of Dental Surgery and Faculty of Surgery were selected at random. In the WACP, Faculty of Pediatrics and Faculty of Laboratory Medicine were selected at random. Twenty resident doctors each were selected using simple random sampling from a list of all resident doctors in the 4 different faculties selected.

In LTH Ogbomosho, 20 resident doctors were selected at random from a list of all resident doctors in the hospital the residents were divided into 2 based similar to how it was described above. Sample size proportional to the population size was done such that 8 medical-based and 12 surgical-based residents were selected using a simple random sampling.

\section{Data Collection and Tools}

Participants were given self-administered questionnaires to fill, which comprised sections on participants sociodemographic data, the CBI, the OLBI, and the MBI (100 copies of MBI-HSS for medical personnel was purchased on August 19, 2020, from mindgarden.com).

\section{Maslach Burnout Inventory Human Services Survey}

The MBI-HSS is a 22-item survey that covers 3 areas: emotional exhaustion (MBI-EE, 9 items), depersonalization (MBI-DP, 5 items), and personal accomplishment (MBI-PA, 8 items). Each question had a 7-point Likert scale response option ranging from 0 , "never" to 6, "everyday" which study participants picked from. The scores of the questions in each subscale were added and considered separately. The permission to use the MBI-HSS was obtained from Mind Garden Incorporation on August 19, 2020.

CBI and OLBI Compared with Maslach Burnout Inventory
Table 2. Summary of participants scores in the various burnout dimensions and their Cronbach's alpha (internal reliability) score

\begin{tabular}{lll}
\hline Variables & Mean (SD) & $\begin{array}{l}\text { Cronbach's alpha } \\
\text { scores }\end{array}$ \\
\hline CPPBO & $47.11(18.14)$ & 0.91 \\
CPWRBO & $43.95(18.07)$ & 0.87 \\
CPPRBO & $26.10(16.19)$ & 0.83 \\
OLBI-E & $2.56(0.44)$ & 0.75 \\
OLBI-DE & $2.36(0.39)$ & 0.70 \\
MBI-EE & $19.47(9.33-22.14)^{*}$ & 0.89 \\
MBI-DP & $4.89(1.34-8.79)^{*}$ & 0.68 \\
MBI-PA & $37.31(6.02)$ & 0.62
\end{tabular}

Maximum score for the Copenhagen dimensions $=100$. Maximum score for the Oldenburg dimensions $=4$. Maximum score for the various Maslach Dimensions: $\mathrm{EE}=54, \mathrm{DP}=30, \mathrm{PA}=48 .{ }^{*}$ Median (IQR).

Copenhagen Burnout Inventory

CBI comprised 19 positively and negatively framed items that cover 3 scales: Personal burnout (CPPBO, 6 items), work-related burnout (CPWRBO, 7 items), and patient-related burnout (CPPRBO, 6 items). Each question has a 5-point Likert scale (never, seldom, sometimes, often, and always) response option which study participants picked from. Before calculating the mean scores for each of the components, the negatively worded questions were reversed where necessary. Weighted scores (percentages) were assigned to each option as follows; never (0), seldom (25), sometimes (50), often (75), and always (100). The score for the set of questions on each of the 3 burnout scales was added and the average of the scores was calculated.

Oldenburg Burnout Inventory

OLBI comprises 16 items positively and negatively framed questions that covers 2 scales, disengagement (OLBI-DE, 8 items), and exhaustion (OLBI-E, 8 items). Response to each question is in the form of a 4-point Likert scale ranging from strongly agrees to strongly disagree. Before calculating mean scores for each of the 2 components, negatively worded items were reversed where necessary, after which an overall score was computed for each scale.

\section{Data Analysis}

Data were analyzed using IBM SPSS version 26. Normality test of each continuous variable was checked using Shapiro-Wilk's test, such that the mean (standard deviation) was reported for normally distributed data and median (interquartile range) for not normally distributed data. The frequency (percentages) of qualitative data was reported. Internal reliability of each of the burnout inventory scales was calculated using Cronbach's alpha. The scales within the 3 burnout tools were correlated with one another to check the construct validity using Pearson's correlation coefficient and principal component analysis. The criterion validity of each scale within the 3 burnout tools was checked using multiple linear regression analysis. The response variable for each model was the score of the various scales (dimensions) within each burnout tool. The significance level was set at $p<0.05$. 
Table 3. Bivariate matrix correlations of the burnout dimensions with one another

\begin{tabular}{lllllllll}
\hline & CPPBO & CPWRBO & CPPRBO & OLBI-E & OLBI-DE & MBI-EE & MBI-DP & MBI-PA \\
\hline CPPBO & 1 & & & & & & & \\
CPWRBO & $0.81^{*}$ & 1 & & & & & & \\
CPPRBO & $0.42^{*}$ & $0.55^{*}$ & 1 & & & & \\
OLBI-E & $0.73^{*}$ & $0.75^{*}$ & $0.37^{*}$ & 1 & & & \\
OLBI-DE & $0.38^{*}$ & $0.44^{*}$ & $0.51^{*}$ & $0.44^{*}$ & 1 & & & \\
MBI-EE & $0.69^{*}$ & $0.79^{*}$ & $0.61^{*}$ & $0.68^{*}$ & $0.58^{*}$ & 1 & & \\
MBI-DP & $0.33^{\dagger}$ & $0.43^{*}$ & $0.49^{*}$ & $0.33^{\dagger}$ & $0.45^{*}$ & $0.55^{*}$ & 1 \\
MBI-PA & -0.18 & -0.20 & -0.17 & $-0.30^{\dagger}$ & $-0.34^{\dagger}$ & $-0.23^{\wedge}$ & $-0.26^{\wedge}$ & 1 \\
\hline
\end{tabular}

${ }^{*} p<0.001 .{ }^{\dagger} p<0.01 .^{\wedge} p<0.05$.

Table 4. Principal component analysis

\begin{tabular}{lll}
\hline & Component 1 (56\%) & Component 2 (14\%) \\
\hline CPWRBO & 0.917 & \\
CPPBO & 0.885 & \\
MBI-EE & 0.822 & 0.375 \\
OLBI-E & 0.776 & \\
CPPRBO & 0.658 & 0.362 \\
MBI-PA & & -0.858 \\
OLBI-DE & & 0.664 \\
MBI-DP & & 0.563 \\
\hline
\end{tabular}

KMO measure of sampling adequacy $=0.831$. Bartlett's Test $X^{2}=$ 355.052, $\mathrm{df}=28$, sig $=0.000$. Extraction method $=$ PCA. Rotation method $=$ Varimax with Kaiser method.

\section{Results}

The results showed that study participants had a median age of 35 (32.5-38.5) years, median of 4 (1-7) years on current job and median of $10(7-11)$ years since graduation from medical school (Table 1). This survey had a $100 \%$ completion rate.

\section{Internal Reliability and Concurrent Validity}

The Cronbach's alpha score was highest in the Copenhagen Personal Burnout scale with a score of 0.91, while the lowest score was in the Maslach Personal Accomplishment 0.62 . Copenhagen burnout inventory dimensions had the highest scores, followed by the Oldenburg burnout dimension scores and finally Maslach dimension (though the EE dimension had a score of 0.888) (Table 2).
Table 5. Multiple regression models predicting the scores of the various burnout dimensions

\begin{tabular}{llll}
\hline & $R$ & $R^{2}$ & $\begin{array}{l}\text { Overall model } \\
\text { significance }(p \text { value) }\end{array}$ \\
\hline CPPBO & 0.38 & 0.15 & $\mathbf{0 . 0 4 5 ^ { * }}$ \\
CPWRBO & 0.39 & 0.15 & $\mathbf{0 . 0 4 *}$ \\
CPPRBO & 0.22 & 0.04 & 0.74 \\
OLBI-E & 0.37 & 0.14 & 0.085 \\
OLBI-DE & 0.23 & 0.05 & 0.714 \\
MBI-EE & 0.25 & 0.06 & 0.55 \\
MBI-DP & 0.15 & 0.02 & 0.967 \\
MBI-PA & 0.32 & 0.11 & 0.192 \\
\hline
\end{tabular}

$* p<0.05$.

\section{Construct Validity (Correlations and PCA)}

Maslach Burnout Inventory-Emotional Exhaustion (MBI-EE) had the highest correlations (very strong positive) with Copenhagen work-related burnout $(r=0.79$, $p<0.001)$, Copenhagen personal burnout $(r=0.69, p<$ $0.001)$, and Oldenburg exhaustion $(r=0.68, p<0.001)$. Maslach depersonalization had weak or moderately strong positive correlations with all the other burnout dimensions (Table 3). MBI-PA had negative correlations with all the other burnout dimensions. The greatest positive correlation was seen between Copenhagen personal burnout and work-related burnout $(r=0.81, p<0.001)$.

Copenhagen Burnout Inventory-Personal Burnout (CPPBO) had a strong positive correlation with Copenhagen Burnout Inventory-Work-Related Burnout (CPWRBO), Oldenburg Burnout Inventory-Exhaustion dimension (OLBI-E), and MBI-EE. CPWRBO had a strong positive correlation with OLBI-E and MBI-EE. OLBI-E had a strong positive correlation with MBI-EE. 
Table 6. Significant predictor variable(s) for predicting CPPBO score

\begin{tabular}{lllll}
\hline & $\begin{array}{l}\text { Regression } \\
\text { coefficient }\end{array}$ & Significance & \multicolumn{2}{l}{$95 \%$ confidence interval } \\
\cline { 3 - 5 } & & lower & upper \\
\hline Specialty & -9.45 & 0.013 & -16.85 & -2.05 \\
\hline
\end{tabular}

$R=0.38 ; R^{2}=0.15 ; p=0.045$.

Table 7. Significant predictor variable(s) for predicting CPWRBO score

\begin{tabular}{lllll}
\hline & \multirow{2}{*}{$\begin{array}{l}\text { Regression } \\
\text { coefficient }\end{array}$} & Significance & \multicolumn{2}{c}{$95 \%$ confidence interval } \\
\cline { 3 - 5 } & & & lower & upper \\
\hline Marital status & -11.44 & 0.008 & -19.86 & -3.03 \\
Specialty & -9.33 & 0.013 & -16.60 & -2.05 \\
\hline
\end{tabular}

$R=0.39 ; R^{2}=0.15 ; p=0.04$.

The goodness-of-fit test (Bartlett's test of sphericity) is significant $(p<0.001)$ which shows the principal component analysis (PCA) is valid (Table 4). The Kaiser-MeyerOlkin (KMO) measure of sampling adequacy is 0.831 , implying the effectiveness of the PCA (Table 4). Two components explained $70 \%$ of the total variation observed with eigen values of at least 1 . After rotation using varimax method, component 1 consisted of CPWRBO, CPPBO, MBI-EE, OLBI-E, and CPPRBO while component 2 was made up of MBI-PA, Maslach depersonalization, and Oldenburg disengagement.

\section{Regression Models (Predictive Validity)}

Multiple linear regression models using least square were computed for each of the dimensions within the 3 burnout tools using age, cadre, specialty, gender, marital status, no of years on current job, and number of years since graduation as independent variables. These independent variables were inputted in each model to predict the scores of the different dimensions of burnout. The response variables in each model were the various burnout dimensions in the 3 burnout tools, thus we had 8 models in all. Only the models predicting CPPBO $(p=0.045)$ and $\mathrm{CPWRBO}(p=$ $0.04)$ scores were significant as shown in Table 5.

Specialty was a significant predictor variable both in models predicting the score of CPPBO and CPWRBO (Tables 6, 7). Resident doctors in surgical specialties had

CBI and OLBI Compared with Maslach Burnout Inventory almost 10 units of lower CPPBO and CPWRBO scores compared to those in medical specialties. Marital status was only a significant predictor variable in predicting the score of CPWRBO (Table 7). Resident doctors who were married had over 11 units reduction in their CPWRBO score compared to those who were single.

\section{Discussion}

This pilot study is important, in that it provides evidence for the use of alternative burnout measures to MBI among resident doctors in Nigeria and by extension possibly other health care workers. This study assessed the internal reliability of the various tools for measuring burnout and the comparativeness of CBI and OLBI to MBI-HSS. The findings from this study showed that the values gotten for the OLBI and MBI-HSS were similar to the findings from other studies among health care workers $[3,27]$. The OLBI scores gotten from our study were similar to that reported by Nwosu et al. on physician's burnout [32], and also followed a similar trend with the OLBI-E being marginally higher than the Oldenburg disengagement in both studies. These similarities could be due to similar working environment as burnout is clearly related to work environment. For this same reason, the findings of higher CBI scores than that reported in other studies among health care workers $[36,37]$ in different socioeconomic environment may not be far-fetched. It could be argued based on our findings that there is an existential moderate level of burnout among resident doctors in Nigeria, though a more generalizable study may be needed to confirm this.

Our findings identified that CBI dimensions (CPPBO and CPWRBO) correlate most with MBI-EE, these 3 dimensions (CPPBO, CPWRBO, and MBI-EE) had the highest internal reliability score $(>0.85)$. CBI measured only a single dimension of burnout which is exhaustion, OLBI measured 2 dimensions (exhaustion and disengagement), while MBI measured 3 dimensions, namely, exhaustion, depersonalization, and reduced personal accomplishment [38]. This study shows all the dimensions in each burnout inventory that measured exhaustion were significantly correlated to one another. This finding was further depicted by the PCA in which all the dimensions of CBI, MBI-EE, and OLBI-E were in component 1 , thus all measuring the same construct. Some authors have argued that exhaustion is perhaps the main dimension of burnout and that personal accomplishment is the weakest burnout dimension in terms of its relationship 
with other variables [27, 39, 40], Kristensen et al. [26] further proposed that PA may not be part of the total concept of burnout. In terms of strict measurement of exhaustion dimension of burnout and its construct validity, the CBI does comparatively as well as the MBI and may even look deeper into the dimensions of exhaustion in terms of the personal, work-related, and patient-related.

The predictive ability of a scale is one of the criteria to determine its validity; our findings showed that CP$\mathrm{PBO}$ and $\mathrm{CPWRBO}$ dimensions of $\mathrm{CBI}$ when adjusting for all the baseline individual characteristics could predict the exhaustion of resident doctors. This finding makes a strong case for the use of CBI and an advantage of the CBI over the exhaustion dimensions of MBI and OLBI among resident doctors. Of note is the insignificance of the patient-related burnout of CBI, while this also measures the exhaustion dimension of burnout, this study finds less correlation with the other 2 dimensions. This perhaps suggests that the burnout felt by resident doctors is much more correlated to the rigors and routine of their work schedule and less correlated to the management of their patients. Work schedule, work environments, personal relationship, and the social life perhaps play a more important role in the impact and perception of burnout than their direct interaction with patients.

This study being a pilot, recruited its participants from just two centers located in the Southwest of Nigeria. It may be difficult to generalize our findings to every resident doctor in Nigeria. Furthermore, we also cannot rule out the effect of self-reporting bias in the outcome of this study. Criticisms trailing the use of MBI has bordered on its temporal and sequential inconsistencies and the tendency for all the questions to be keyed in the same direction [26]. While the initial argument may not be valid for CBI, the latter is also largely true for CBI. All the questions on the CBI except one are keyed in the same direction, whereas the OLBI has both negatively and positively worded questions [38]. Though findings from our study suggest the superiority of CBI over OLBI in terms of the criterion (predictive) and construct (convergent) validity, the direction of the wording of the questionnaire could affect response and bias results [41]. With a larger generalizable sample size, and modification of the direction of the wording of CBI, a more robust comparison between CBI and OLBI assessment of burnout among residents could be carried out.

\section{Conclusion}

While this study is a pilot, it clearly shows that CBI dimensions and OLBI have high internal consistency and high reliability among the subset of resident doctors recruited into this study and can be used as valid alternative to $\mathrm{MBI}$ among resident doctors. This study also suggests the CBI even though measures only the exhaustion dimension of burnout, has a better criterion (concurrent and predictive) and construct (convergent) validity than OLBI and MBI, and may be a suitable no-cost alternative to the copyrighted MBI. We anticipate this finding would have impact on the output of the greater data from the CHARTING II study currently ongoing [34].

\section{Acknowledgments}

We like to appreciate Miss Iyanu Adufe for her role in the clerical and data entry duties during this study. We equally appreciate The National Executive Council of NARD, Research Collaboration Network advisors and all the participants for their cooperation during recruitment and data collection for this study.

\section{Statement of Ethics}

Ethical approval was gotten for this pilot study as part of CHARTING II study from Oyo State Research Ethics Review Committee (AD13/479/2039 ${ }^{\mathrm{B}}$ ) and National Health Research Ethics Committee of Nigeria (NHREC/01/01/2007-06/08/2020B). All participants recruited into this study gave written informed consent.

\section{Conflict of Interest Statement}

All authors are members of NARD except the last 2 authors as at the time of this study; however, the study was independently conducted and reported. NARD played only a funder's role.

\section{Funding Sources}

This work was supported by the Nigerian Association of Resident Doctors (NARD) under funding for Research \& Statistics Committee (Grant No. 0002).

\section{Author Contributions}

O.A. ${ }^{1}$, O.O., ${ }^{1}$ and H.O. contributed to conceptualization; O.O. ${ }^{1}$, H.O., and O.A. ${ }^{1}$ contributed to methodology; O.O. ${ }^{1}$, H.O., T.O., S.O., O.O. ${ }^{2}$, O.S., O.A. ${ }^{2}$, and T.A. contributed to data collec-
Ogunsuji et al. 
tion; O.O. ${ }^{1}$. contributed to data analysis; O.A. ${ }^{1}$ contributed to administration; O.O. ${ }^{1}$, H.O., and O.A. ${ }^{1}$ contributed to writing of initial draft; all authors contributed to writing, review, and editing of final draft; all the authors listed were involved in the writing of the final draft, and all the authors agreed to be responsible for the outcome of the manuscript.

\section{Data Availability Statement}

Data that support the finding of this article are not publicly available as it contains information that could compromise the privacy of research participants but are available from the corresponding author (O.A.) upon reasonable request.

\section{References}

1 World Health Organization. International classification of diseases for mortality and morbidity statistics. 11th ed. World Health Organization; 2018.

2 Maslach C, Jackson SE. The measurement of experienced burnout. J Organ Behav. 1981;2(2): 99-113.

3 Maslach C, Jackson SE, Leiter MP, Schaufeli WB, Schwab RL. Maslach burnout inventory. Palo Alto, CA: Consulting Psychologists Press; 1986.

4 Schaufeli WB, Van Dierendonck D. The construct validity of two burnout measures. J Organ Behav. 1993;14(7):631-47.

5 Leiter MP, Durup J. The discriminant validity of burnout and depression: a confirmatory factor analytic study. Anxiety Stress Coping. 1994;7(4): 357-73.

6 Hallberg UE, Sverke M. Construct validity of the Maslach burnout inventory: two Swedish health care samples. Eur J Psychol Assess. 2004;20(4): 320-38.

7 Coker A, Omoluabi P. Validation of Maslach burnout inventory. IFE PsychologIA. 2009; 17(1):231-42

$8 \mathrm{Hu}$ Q, Schaufeli WB. The factorial validity of the Maslach burnout inventory-student survey in China. Psychol Rep. 2009;105(2):394-408.

9 Lasebikan VO, Oyetunde MO. Burnout among nurses in a Nigerian general hospital: prevalence and associated factors. ISRN Nurs. 2012;2012: 402157-6.

10 Doulougeri K, Georganta K, Montgomery A. "Diagnosing" burnout among healthcare professionals: can we find consensus? Cogent Med. 2016;3(1):1237605.

11 Ibikunle P, Amah E, Useh U. Prevalence and pattern of burnout syndrome among healthcare professionals in a university teaching hospital. Trop J Med Res. 2016;19(2):144-4.

12 Montiel-Company JM, Subirats-Roig C, FloresMartí P, Bellot-Arcís C, Almerich-Silla JM. Validation of the Maslach burnout inventory-human services survey for estimating burnout in dental students. J Dent Educ. 2016;80(11):1368-75.

13 Wickramasinghe ND, Dissanayake DS, Abeywardena GS. Clinical validity and diagnostic accuracy of the Maslach burnout inventory-student survey in Sri Lanka. Health Qual Life Outcomes. 2018;16(1):220-9.

14 Ogundipe OA, Olagunju AT, Lasebikan VO, Coker AO. Burnout among doctors in residency training in a tertiary hospital. Asian J Psychiatr. 2014;10:27-32.

15 Okwaraji FE, Aguwa EN. Burnout and psychological distress among nurses in a Nigerian ter- tiary health institution. Afr Health Sci. 2014; 14(1):237-45.

16 Rotifa S, Eguvbe A, Ofili A, Adeleye O. Likelihood of psychological disorder and burnout among doctors and nurses in tertiary health facilities in Bayelsa state, South-South Nigeria. J Prim Health Care. 2018;2(2):13.

17 Dubale BW, Friedman LE, Chemali Z, Denninger JW, Mehta DH, Alem A, et al. Systematic review of burnout among healthcare providers in Sub-Saharan Africa. BMC Public Health. 2019;19(1):1-20.

18 Ogunsuji O, Adebayo O, Olaopa O, Efuntoye O, Agbogidi J, Kanmodi K, et al. Burnout among Nigerian doctors: a systematic review. Niger Med Pract. 2019;76(1-3):24-9.

19 Ozumba L, Alabere I. Burnout among doctors and nurses at university of Port Harcourt teaching hospital, South-South Nigeria. Arch Med Health Sci. 2019;7(1):61.

20 Ogboghodo EO, Edema OM. Assessment of burnout amongst resident doctors in Benin City, Edo State, Nigeria. Niger Postgrad Med J. 2020; 27(3):215.

21 Chin RWA, Chua YY, Chu MN, Mahadi NF, Wong MS, Yusoff MS, et al. Investigating validity evidence of the Malay translation of the Copenhagen Burnout Inventory. J Taibah Univ Med Sci. 2018;13(1):1-9.

22 Walters JE, Brown AR, Jones AE. Use of the Copenhagen Burnout Inventory with social workers: a confirmatory factor analysis. Hum Serv Organ Manag Leadersh Gov. 2018;42(5):43756.

23 Sinval J, Queirós C, Pasian S, Marôco J. Transcultural adaptation of the Oldenburg burnout inventory (OLBI) for Brazil and Portugal. Front Psychol. 2019;10:338.

24 Demerouti Evangelia, Bakker Arnold, Nachreiner Friedhelm, Schaufeli Wilmar. The job demands-resources model of burnout. Journal of Applied psychology. 2001;86(3):499-512. https: //doi/10.1037/0021-9010.86.3.499.

25 Halbesleben JRB, Demerouti E. The construct validity of an alternative measure of burnout: investigating the English translation of the Oldenburg Burnout Inventory. Work Stress. 2005; 19(3):208-20.

26 Kristensen TS, Borritz M, Villadsen E, Christensen KB. The Copenhagen Burnout Inventory: a new tool for the assessment of burnout. Work Stress. 2005;19(3):192-207.

27 Demerouti E, Bakker AB. The Oldenburg Burnout Inventory: a good alternative to measure burnout and engagement. In: Halbesleben JRB, editor. Handbook of stress and burnout in health care. Nova Science Publishers; 2008. p. 65-78.

28 Demerouti E, Mostert K, Bakker AB. Burnout and work engagement: a thorough investigation of the independency of both constructs. J Occup Health Psychol. 2010;15(3):209.

29 Campos JADB, Carlotto MS, Marôco J. Copenhagen Burnout Inventory-student version: adaptation and transcultural validation for Portugal and Brazil. Psicol Reflex Crít. 2013;26(1): 87-97.

30 Ruiz EM, Gómez-Quintero HB, Lluis SM. Validation of the Copenhagen burnout inventory to assess professional burnout in Spain. Rev Esp Salud Publica. 2013;87(2):165-79.

31 Tipa RO, Tudose C, Pucarea VL. Measuring burnout among psychiatric residents using the Oldenburg Burnout Inventory (OLBI) instrument. J Med Life. 2019;12(4):354.

32 Nwosu AD, Ossai EN, Mba UC, Anikwe I, Ewah R, Obande BO, et al. Physician burnout in Nigeria: a multicentre, cross-sectional study. BMC Health Serv Res. 2020;20(1):1-9.

33 Kanmodi K, Adebayo O, Efuntoye O, Ogunsuji O, Buowari DY, Grillo E, et al. Challenges of residency training and early career doctors in Nigeria study. Niger J Med. 2019;28(2):198205.

34 Eze UA, Tolani MA, Adeniyi MA, Ogbonna VI, Isokariari O, Igbokwe MC, et al. Challenges of residency training and early career doctors in Nigeria phase II: update on objectives, design, and rationale of study. Niger J Med. 2020;29(4):714-9.

35 Okonofua F. Postgraduate medical education in Nigeria: past, present, and future. Trop J Obstet Gynaecol. 2018;35(1):1-13.

36 Winwood PC, Winefield AH. Comparing two measures of burnout among dentists in Australia. Int J Stress Manag. 2004;11(3):282.

37 Borritz M, Rugulies R, Bjorner JB, Villadsen E, Mikkelsen OA, Kristensen TS. Burnout among employees in human service work: design and baseline findings of the PUMA study. Scand J Public Health. 2006;34(1):49-58.

38 Rothenberger DA. Physician burnout and wellbeing: a systematic review and framework for action. Dis Colon Rectum. 2017;60(6):567-76.

39 Lee RT, Ashforth BE. A meta-analytic examination of the correlates of the three dimensions of job burnout. J Appl Psychol. 1996;81(2):123.

40 Schaufeli W, Enzmann D. The burnout companion to study and practice: a critical analysis. CRC Press; 1998.

41 Bowling A. Research methods in health: investigating health and health services. Maidenhead, UK: McGraw-Hill Education; 2014. 